Introduction Hepatitis B (HBV) infection is a major cause of chronic liver disease, which is a leading cause of death worldwide. Despite differing epidemiology, HBV infection shares routes of transmission with HIV infection. Therefore we explored factors associated with those infections among highrisk populations in Peru.

Methods Men who have sex with men (MSM) and transwomen (TW) recruited from two STI clinics completed a behavioural survey on alcohol use and sexual risk-taking in the last 3 months and received HIV testing. An AUDIT score $\geq 17$ determined the presence of a severe alcohol use disorder. Active hepatitis B infection was determined by detection of HBsAg using EIA UMELISA and HBsAg confirmatory testing (Tecnosuma, Cuba). Self-collected rectal swabs were used to diagnose gonorrhoea/ chlamydia by NAAT. Prevalence ratios (PR) were calculated using Poisson regression.

Results Among $310 \mathrm{MSM}$ and 89 TW (median age 30 years, IQR: $18-58), 5 \%(20 / 399)$ had active HBV infection. Of the 20 participants with active HBV infection, 50\% were HIV coinfected which was significantly more than the HIV prevalence among HBsAg negatives $(30 \%$, p-value $=0.053)$. Rates of recent condomless anal intercourse were similar by HBV infection status $(68 \%$ among those with active infection vs. $74 \%$ among HBsAg negatives $\mathrm{p}=0.557$ ). Rectal STI prevalence was $40 \%$ among participants with active HBV infection vs. 20\% among $\mathrm{HBsAg}$ negative participants. ( $\mathrm{p}$-value $=0.034$ ). In multivariable regression active $\mathrm{HBV}$ infection was associated with severe alcohol use disorders $(\mathrm{aPR}=2.54$, $\mathrm{p}$-value $=0.008)$ and HIV diagnosis $(\mathrm{aPR}=2.56$, $\mathrm{p}$-value $=0.009)$.

Conclusions In the context of South America's low carrier rate, the prevalence of active HBV infection among this Peruvian sample is high. Our findings emphasise the importance of revising national HBV screening and vaccination guidelines to include HIV-infected individuals and heavy drinkers since both conditions may accelerate liver disease. Educational campaigns to encourage transmission prevention are also needed since condomless anal intercourse was common in this cohort.

Disclosure of interest statement This data for this abstract was obtained from an NIH-funded study (1R01AI099727). Hepatitis tests were donated however the donating company did not contribute to the conception of this study or participate in the analysis/interpretation of the data in this abstract.

\section{P11.22 HEPATITIS C INFECTIONS AMONG HIGH-RISK MEN WHO HAVE SEX WITH MEN AND TRANSGENDER WOMEN IN LIMA, PERU}

${ }^{1} \mathrm{MC}$ Herrera*, ${ }^{1} \mathrm{KA}$ Konda, ${ }^{2} \mathrm{SR}$ Leon, ${ }^{3} \mathrm{P}$ Wong, ${ }^{4} \mathrm{~B}$ Brown, ${ }^{2} \mathrm{CF}$ Caceres, ${ }^{1} \mathrm{JD}$ Klausner. ${ }^{1}$ Division of Infectious Diseases, UCLA David Geffen School of Medicine, Los Angeles, California, USA; ${ }^{2}$ Laboratory of Sexual Health, and Unit of Health, Sexuality and Human Development, Universidad Peruana Cayetano Heredia, Lima, Peru; ${ }^{3}$ nstituto Peruano de Investigaciones en Biotecnología, Lima, Peru; ${ }^{4}$ Department of Population Health \& Disease Prevention, UC Irvine, IIvine, California, USA

\subsection{6/sextrans-2015-052270.470}

Introduction Disparate findings persist in the literature regarding the sexual transmission of hepatitis $\mathrm{C}$ virus (HCV) and the role of HIV/other STIs. Therefore we analysed the epidemiology and risk factors for HCV infection among a non-intravenous drug using, sexually active population in Peru.

Methods High-risk men who have sex with men (MSM) and transwomen (TW) recruited from two STI clinics completed a behavioural survey on substance use and sexual risk taking in the last 3 months. Hepatitis $\mathrm{C}$ diagnosis was determined by detection of Anti-HCV antibodies using EIA UMELISA PLUS (Specificity: $99.93 \%$ Sensitivity: 100\% Tecnosuma, Cuba). In addition to HIV testing, STI screening included rectal gonorrhoea/chlamydia (NAAT) and syphilis (RPR titer $\geq 1: 16$ ). Chi-square tests were used to compare differences in characteristics between HCVinfected and negative individuals.

Results Among 310 MSM and 89 TW (median age30 years, IQR: 18-58), 4\% (15/399) were HCV-infected. Recent condomless anal intercourse was reported by $76 \%$ of the sample (292/ 393). Although more condomless anal intercourse was reported among HCV-infected vs. negative individuals (93\% vs.74\%) the difference was not significant $(\mathrm{p}=0.107)$. Illicit substance use for the cohort was low with $13 \%$ reporting recent cocaine use, which also was not significantly different among HCV-infected vs. negative individuals $(27 \%$ vs. $13 \%, \mathrm{p}=0.120)$. HIV positivity was $31 \%$ for the sample $(124 / 401)$ and was associated with HCV infection vs. non-infection (53\% vs. $30 \%, \mathrm{p}=0.054)$. When comparing HCV-infected to negative individuals, neither rectal STI diagnosis $(27 \%$ vs. $20 \%, \mathrm{p}=0.55)$ nor recent syphilis diagnosis ( $47 \%$ vs. $36 \%, \mathrm{p}=0.41)$ were significantly associated. Conclusion The findings for this cohort in Peru did uncover an association between HCV infection and concurrent HIV infection however such associations for sexual risk behaviours and other STI diagnoses were not found. Prospective data documenting HCV seroconversion among a larger sample is needed to better understand the contribution of sexual transmission to $\mathrm{HCV}$ infection.

Disclosure of interest statement This data for this abstract was obtained from an NIH-funded study (1R01AI099727). Hepatitis tests were donated however the donating company did not contribute to the conception of this study or participate in the analysis/interpretation of the data in this abstract.

\section{P11.23 EVALUATION OF HEPATITIS C VIRUS IN THE RECTUM OF HIV-INFECTED MEN WHO HAVE SEX WITH MEN}

${ }^{1} \mathrm{~A}$ Foster*, ${ }^{2} \mathrm{M}$ Gaisa, ${ }^{1} \mathrm{~S}$ Turner, ${ }^{1} \mathrm{~T}$ Morey, ${ }^{2} \mathrm{~K}$ Jacobson, ${ }^{2} \mathrm{D}$ Fierer. ${ }^{1}$ James Cook University School of Medicine; ${ }^{2}$ Icahn School of Medicine at Mount Sinai

\subsection{6/sextrans-2015-052270.471}

Introduction An epidemic of Hepatitis C Virus (HCV) infection is occurring in HIV-infected men who have sex with men (MSM). Epidemiological studies suggest that sexual transmission is fueling the epidemic. Blood and semen have been considered as potential mechanisms of transmission, but there are still transmission circumstances that remain unexplained. Nothing is known about the presence of $\mathrm{HCV}$ in the rectal fluid of HCVinfected MSM.

Methods Written informed consent was obtained from 45 HIVinfected MSM with HCV infection. A moistened swab was inserted atraumatically into the rectum, placed into transport medium, vortexed, and the supernatant analysed for HCV using AmpiPrep/COBAS TaqMan HCV test (Roche Diagnostics), lower limit of quantification $43 \mathrm{IU} / \mathrm{mL}$, lower limit of detection $7 \mathrm{IU} / \mathrm{mL}$. Results Successful virological analysis was performed on 43 rectal swabs. HCV was detected in 20 out of $43(47 \%)$ specimens. The HCV viral load (VL) in the rectal fluid ranged from $<2.92$ - $5.52 \log _{10} \mathrm{IU} / \mathrm{mL}$. Rectal HCV detection was associated with 
serum HCV VL $>5 \log _{10} \mathrm{IU} / \mathrm{mL}(\mathrm{p}=0.011)$. The magnitude of paired rectal and serum HCV VLs were strongly correlated (correlation coefficient $0.688, \mathrm{p}<0.001$ ). Based on the median quantifiable rectal VL in the absence of visible blood, the surface of an average human penis would be exposed to 2,496 IU of HCV for the duration of anal intercourse.

Conclusion This study provides the first documentation of the presence of $\mathrm{HCV}$ in non-blood rectal fluid. It is plausible that the combination of bathing of an inserted penis in rectal HCV, with the attendant friction of anal intercourse, could result in penetration of HCV into the inserted penis. The protection to the penis afforded by a condom would logically mitigate the risk of transmission by this mechanism. This study should inform public health policy concerning the primary prevention of sexually transmitted HCV.

Disclosure of interest statement There are no competing or financial interests to disclose.

\section{P11.24 LANDSCAPE OF HSV2 AND HIV INFECTIONS AMONG MSMS IN MOROCCO: RESULTS FROM A RESPONDENT DRIVEN SAMPLING SURVEY}

${ }^{1} \mathrm{H}$ Oumzil ${ }^{*},{ }^{1} Y$ Ikken, ${ }^{1} \mid$ Belbacha, ${ }^{1} \mathrm{H}$ Eloudyi, 'S Lemrabet, ${ }^{1} \mathrm{O}$ Bennani, ${ }^{1} \mathrm{R}$ Mengad, ${ }^{2} \mathrm{~A}$ Latifi, ${ }^{3} \mathrm{H}$ Rhilani, ${ }^{3} \mathrm{~K}$ Alami, 'E Elharti. ${ }^{1}$ National Referral Laboratory for HIV, National Institute of Hygiene, Rabat. Morocco; ${ }^{2}$ National Aids Program, Ministry of Health. Morocco; ${ }^{3}$ UNAIDS, Rabat, Morocco

\subsection{6/sextrans-2015-052270.472}

Introduction Over the past two decades, Herpes simplex virus type 2 (HSV2) became the most common cause of genital ulcer in the developed and the developing countries. It represents a risk factor for the acquisition and transmission of other sexually transmitted diseases such as HIV.

With regard to the lack of reports about HSV2 prevalence among Men having Sex with Men (MSMs) in Morocco, this study was conducted to determine the prevalence of the virus among MSMs associated to the risk behaviour.

Methods MSMs from two regions (Marrakech and Agadir) were recruited using Respondent-driven sampling, a chain referral sampling approach. Blood samples collected from 669 recruits were assessed for IgG antibody of HSV2 using ELISA method. Positive samples were tested for IgM to assess recent infection. HIV diagnosis was performed using ELISA test. Reactive samples were confirmed using western bot. Discordant tests were confirmed using PCR. Socio-demographic informations and behavioural data were collected to assess the risk factors associated with infections.

Results The results showed an overall HSV2 sero prevalence of 23\%, and $4.2 \%$ for HIV1 infection. Co-infection with HSV2 among HIV positive recruits was estimated to 50\%. Among HSV 2 reactive recruits, 7\% were positive for HSV 2 IgM, which testifies a recent infection.

Only 22.4\% of MSMs consistently used condoms with male partners and 35\% had never been tested for HIV.

A Risky sexual behaviour and a lack of knowledge about STIs and HIV were the main driver of this situation.

Conclusion Findings suggest continuing STIs risk among MSM in Morocco and a need to strengthen prevention and testing.

\section{P11.25 TIMOR-LESTE NATIONAL SIZE ESTIMATES AND BEHAVIOURAL DATA FOR KEY POPULATIONS AT RISK OF HIV AND OTHER STIS}

${ }^{1} \mathrm{H}_{\text {Jose }}{ }^{*},{ }^{2} \mathrm{P}$ Rawstorne, ${ }^{2} \mathrm{~B}$ Rahman, ${ }^{3} \mathrm{~K}$ Dolan. ${ }^{1}$ Australasian Society for HIV Medicine; ${ }^{2}$ School of Public Health and Community Medicine, UNSW; ${ }^{3}$ National Centre for Drug and Alcohol Research

10.1136/sextrans-2015-052270.473

Introduction The Timor-Leste HIV/STI response is largely focused on the key populations of sex workers, men who have sex with men and transgender (MSM\&TG) and people who use and/or inject drugs (PWUD/PWID). With no prior national size estimation (NSE), a NSE was undertaken to inform HIV/STI programming for these population groups. Behavioural surveying of FSW and MSM\&TG in Dili was also completed.

Methods To improve reliability, several size estimation methods were used: Network Scale-Up; Service Multiplier; Successive Sampling - Size; and 'Wisdom of the Crowd'. Estimates were cross-referenced with service records, estimates from comparable settings and key informant estimates. Plausible estimates were combined and extrapolated to non-assessed districts. A consensus process with key stakeholders was undertaken for validation. For behavioural data, a respondent-driven sampling (RDS)-based survey of FSW and MSM\&TG was conducted in the capital of Dili. Results

1. National size estimates

- FSW: 1688 (95\% CI: 1333, 2044); 0.71\% females aged $15-49$

- MSM\&TG: 8703 (95\% CI: 7821, 9,585); 2.76\% males aged $15+$

- PWUD: 388 (95\% CI: 208, 787); 0.07\% adults aged 15-64

- PWID: 53 (95\% CI: 10, 127); <0.01\% adults aged 15-64

\section{Behaviour}

A total of 125 FSW and 229 MSM\&TG participated in the Dili behavioural survey. Service interaction was mixed, with $67.8 \%$ (95\% CI: $55.7 \%, 79.0 \%, \mathrm{n}=91$ ) of FSW and 51.0\% (95\% CI: $40.6 \%, 61.1 \%, \mathrm{n}=124)$ of MSM\&TG reporting ever having had an HIV test. About one-quarter of both FSW (25.7\%, 95\% CI: $16.1 \%, 34.3 \%, \mathrm{n}=39)$ and MSM\&TG $(26.1 \%, 95 \% \mathrm{CI}$ : $18.8 \%, 33.5 \%, \mathrm{n}=51$ ) reported always using condoms with their client/male sexual partner respectively. Reported illicit drug use among both populations was extremely low.

Conclusion Estimates produced were deemed plausible. The comparably low national PWUD/PWID estimates reflected an extremely low current prevalence of illicit drug use in the country. Behavioural data indicated ongoing HIV/STI risk factors for FSW and MSM\&TG.

Disclosure of interest statement This project received funding from the Ministry of Health Timor-Leste under a grant from the Global Fund to fight AIDS, Tuberculosis and Malaria. No pharmaceutical grants were received in the development of this study.

\section{P11.26 THE SOCIAL AND SEXUAL DYNAMIC BETWEEN GAY MEN/TRANSGENDER PEOPLE AND THEIR STRAIGHT- IDENTIFYING MALE PARTNERS IN TIMOR-LESTE}

${ }^{1} \mathrm{H}$ Jose*, ${ }^{2} \mathrm{P}$ Rawstorne, ${ }^{3} \mathrm{P}$ Gonzaga, ${ }^{2} \mathrm{~S}$ Nathan. ${ }^{1}$ Australasian Society for HIV Medicine; ${ }^{2} \mathrm{School}$ of Public Health and Community Medicine, UNSW; ${ }^{3} \mathrm{~N} / \mathrm{a}$

10.1136/sextrans-2015-052270.474 